\title{
Leishmania amazonensis promastigotes in 3D Collagen I culture: an in vitro physiological environment for the study of extracellular matrix and host cell interactions.
}

Leishmania amazonensis is the causative agent of American cutaneous leishmaniasis, an important neglected tropical disease. Once Leishmania amazonensis is inoculated into the human host, promastigotes are exposed to the extracellular matrix (ECM) of the dermis. However, little is known about the interaction between the ECM and Leishmania promastigotes. In this study we established L. amazonensis promastigote culture in a threedimensional (3D) environment mainly composed of Collagen I (COL I). This 3D culture recreates in vitro some aspects of the human host infection site, enabling the study of the interaction mechanisms of $L$. amazonensis with the host ECM. Promastigotes exhibited "freeze and run" migration in the 3D COL I matrix, which is completely different from the conventional in vitro swimming mode of migration. Moreover, L. amazonensis promastigotes were able to invade, migrate inside, and remodel the 3D COL I matrix. Promastigote transmatrix invasion and the freeze and run migration mode were also observed when macrophages were present in the matrix. At least two classes of proteases, metallo- and cysteine proteases, are involved in the 3D COL I matrix degradation caused by Leishmania. Treatment with a mixture of protease inhibitors significantly reduced promastigote invasion and migration through this matrix. Together our results demonstrate that $L$. amazonensis promastigotes release proteases and actively remodel their 3D environment, facilitating their migration. This raises the possibility that promastigotes actively interact with their $3 D$ environment during the search for their cellular "home" - macrophages. Supporting this hypothesis, promastigotes migrated faster than macrophages in a novel 3D co-culture model. 
1 Debora B. Petropolis ${ }^{1,2}$; Juliany C. F. Rodrigues ${ }^{1,3,4,5}$; Nathan B. Viana ${ }^{6,7}$; Bruno Pontes ${ }^{6}$; Camila F.

2 A. Pereira ${ }^{1}$; Fernando C. Silva-Filho ${ }^{1,8}$

3 Corresponding author: Debora B. Petropolis

4 Address: 28 rue du Dr. Roux, Paris, 75015

5 Email address: dpetropo@pasteur.fr

6 Phone number. +330140613152

$7{ }^{1}$ Instituto de Biofisica Carlos Chagas Filho; Universidade Federal do Rio de Janeiro, Rio de 8 Janeiro, Brazil; ${ }^{2}$ Institut Pasteur, Paris, France; ${ }^{3}$ Núcleo Multidisciplinar de Pesquisa UFRJ9 Xerém (NUMPEX-BIO), Polo Avançado de Xerém, Universidade Federal do Rio de Janeiro, 10 Brazil; ${ }^{4}$ Instituto Nacional de Ciência e Tecnologia de Biologia Estrutural e Bioimagem, Brazil; ${ }^{5}$ 11 Instituto Nacional de Metrologia, Qualidade e Tecnologia, Inmetro, Brazil; ${ }^{6}$ LPO-COPEA, 12 Instituto de Ciências Biomédicas, Universidade Federal do Rio de Janeiro, Brazil; ${ }^{7}$ Instituto de 13 Física; Universidade Federal do Rio de Janeiro; Rio de Janeiro, Brazil. ${ }^{8}$ Universidade Federal do 14 Norte Fluminense, Campos, Brazil 
Introduction

16 The promastigote form of Leishmania amazonensis, causative agent of American cutaneous

17 leishmaniasis, (Barral et al. 1991; Murray et al. 2005) is transmitted through the bite of infected

18 sand flies from the genus Lutzomyia (Kaye \& Scott 2011; Neuber 2008). Once inoculated, the

19 promastigotes are exposed to the dermis microenvironment, which is composed of extracellular 20 matrix (ECM) proteins organized in a fibrilar network (Rogers 2012). Because L. amazonensis is

21 an intracellular parasite that only proliferates inside a cellular host in the mammalian host, its

22 interaction with the host ECM has been neglected. However, in order to establish an intracellular

23 infection, the promastigote form must overcome the obstacles presented by the dermis ECM

24 (Chang \& McGwire 2002). Except for broad ECM alterations during experimental Leishmania

25 infection (Abreu-Silva et al. 2004; Melo et al. 2009; Silva-Almeida et al. 2012a) little is known

26 about the interaction of L. amazonensis with the host ECM (Kulkarni et al. 2008; McGwire et al.

27 2003). Although long viewed only as a supportive structure, the ECM is an essential part of the

28 cell's milieu that, through direct or indirect means, regulates almost all cellular behavior ( $\underline{\text { Hynes }}$

29 2009), including inflammatory signaling (Larsen et al. 2006).

30 The glycoprotein gp63 presented on parasite surface is a broad-spectrum zinc-dependent 31 metalloprotease that works as an important virulence factor during Leishmania infection

32 (McMaster et al. 1994; Silva-Almeida et al. 2012a; Yao et al. 2002). gp63 can degrade ECM

33 components such as fibronectin and collagen IV and seems to enhance L. amazonensis migration

34 in Matrigel, a commercial 3D gelatin of basement membrane ECM proteins (Kulkarni et al. 2008;

35 McGwire et al. 2003). However, collagen I (COL I) is the main component of the dermis ECM,

36 and for the moment little is known about the interaction of L. amazonensis with COL I rich

37 environments (Lira et al. 1997).

38 Here, we introduce a new model for culture of L. amazonensis promastigotes in an in vitro 3D

39 COL I matrix, which represents a more physiological in vitro culture because it better mimics 
40 many extracellular aspects of the Leishmania environment in the human host. The objective of

41 this work was to characterize L. amazonensis invasion, migration, and matrix remodeling in 3D

42 COL I matrices, focusing on the functions of Leishmania proteases. The establishment of this

43 model enables evaluation of the mechanisms of Leishmania interaction with host cells in a 3D

44 environment.

\section{Methods}

\section{Ethics Statement}

47 The use of animal models was approved by the Ethics Committee for Animal Experimentation of

48 the Health Sciences Centre, Federal University of Rio de Janeiro (Protocols n. IBCCF 096/097),

49 according to the Brazilian federal law. All animals received humane care in compliance with the

50 "Principles of Laboratory Animal Care" formulated by the National Society for Medical Research

51 and the "Guide for the Care and Use of Laboratory Animals" prepared by the National Academy

52 of Sciences, USA.

\section{Leishmania amazonensis promastigotes culture}

54 The MHOM/BR/75/Josefa strain of L. amazonensis used in this study is an anonymized strain

55 that was isolated in 1975 from a patient with diffuse cutaneous leishmaniasis by Dr. Cesar A.

56 Cuba-Cuba (Brasilia University, Brazil) and kindly provided by the Leishmania Collection of the

57 Instituto Oswaldo Cruz (Code IOCL 0071 - FIOCRUZ). It has been maintained by BALB/c

58 footpad inoculation. Amastigote forms were obtained from these mice, and transformed into

59 promastigotes that were axenically cultured in Warren's medium (brain and heart infusion with

$6020 \mu \mathrm{g} / \mathrm{ml}$ hemin and $10 \mu \mathrm{g} / \mathrm{ml}$ folic acid) supplemented with $10 \%$ fetal bovine serum at $25^{\circ} \mathrm{C}$.

61 Stationary-phase promastigotes were obtained from 5- to 6-day-old cultures and used throughout. 


\section{L. amazonensis promastigote cultivation on 3D Collagen I matrices}

63 Rat tail extracted COL I solution (Rajan et al. 2007) was diluted with 5-times concentrated

64 DMEM media and completed to the desired density $(1.5$ or $3.0 \mathrm{mg} / \mathrm{mL})$ with DMEM. $0.1 \mathrm{M}$

$65 \mathrm{NaOH}$ was used to neutralize the solution $\mathrm{pH} \cdot 10^{7}$ promastigotes were mixed with $1 \mathrm{~mL}$ of 66 diluted and neutralized COL I solution and incubated at $37^{\circ} \mathrm{C}$ for a 1 hour polymerization. After

67 complete polymerization, serum-free RPMI medium was added to the top of the matrices to feed

68 the cells and hydrate the COL I matrix. The culture was then left at $27^{\circ} \mathrm{C}$ for no more than 72

69 hours. Viability was measured using a fluorescent Live/Dead assay (Invitrogen, L3224) and only

70 samples with viability scores higher than $90 \%$ were used.

\section{Scanning Electron Microscopy}

72 COL I matrix samples cultivated with L. amazonensis promastigotes were fixed with $2.5 \%$ 73 glutaraldehyde in $0.1 \mathrm{M}$ cacodylate buffer $(\mathrm{pH}$ 7.2) for 1 hour and then washed overnight with 74 PBS. After fixation, promastigotes in the COL I matrix were postfixed for 30 min in a solution 75 containing $1 \% \mathrm{OsO}_{4}, 1.25 \%$ potassium ferrocyanide and $5 \mathrm{mM} \mathrm{CaCl}_{2}$ in 0.1 cacodylate buffer, 76 washed in the same buffer and then dehydrated in an ethanol series from $30 \%$ to $100 \%$. Finally, 77 samples were critical point dried, coated with a thin gold layer in a Balzers gold sputtering 78 system, and observed in a FEI-Quanta scanning electron microscope.

\section{Protease inhibitors}

80 Metalloprotease inhibitors included $200 \mathrm{nM}$ Marimastat or $5 \mathrm{mM}$ o-phenantroline, cysteine 81 protease inhibitors included $20 \mathrm{ng} / \mathrm{mL}$ Cystatin or $100 \mu \mathrm{M}$ of trans-epoxysuccinyl-L-

82 leucylamido-(4-Guanidino)butane (E-64) and the serine protease inhibitor used was $1 \mathrm{mM} 4-(2-$ 83 aminoethyl)-benzenesulfonylfluoride (AEBSF), (all protease inhibitors were purchased from 84 Sigma-Aldrich). Protease inhibitors (PI) mix was used in some experiments. The PI mix used was 
composed of AEBSF, E-64, Cystatin and Marimastat.

86

87

88

89

90

91

92

93

94

95

96

97

98

99

100

101

102

103

104

105

106

\section{Migration assay}

The L. amazonensis promastigote migration was characterized for all experimental conditions. Samples were placed in a culture chamber, with controlled temperature $\left(27^{\circ} \mathrm{C}\right)$, adapted to an inverted microscope Nikon Eclipse TE 300 (Nikon, Melville, NY). Brightfield images were captured for 5 minutes with a Hamamatsu C2400 CCD camera (Hamamatsu, Japan) and digitalized by a SCION FG7 frame grabber (Scion Corporation, Torrance, CA) using a frame rate of 2 frames/s. The process was repeated every 24 hours for a total period of 72 hours. For each movie 15 different cells were marked with black dots. The distance covered by the black dot trajectories were then obtained by image analysis using the ImageJ software (National Institutes of Health, USA) and the indirect promastigote migration rates were determined by dividing the distance covered $(\mu \mathrm{m})$ by the time in seconds $(\mathrm{sec})$. Three different movies were made and analyzed for each condition.

\section{Transmatrix migration (invasion) assay}

For the transmatrix migration assay, the COL I matrix was previously prepared without $L$. amazonensis in a 96 well plate. Then, $100 \mu \mathrm{L}$ serum free RPMI media containing $10^{6}$ promastigotes was added to the top of a $150 \mu \mathrm{L}$ polymerized matrix. After 48 hours, the RPMI media was washed out and the samples were fixed and processed for histology. The quantity of promastigotes inside the matrix (complete transmatrix process) and the distance migrated from the top to the bottom of the COL I matrix (transmatrix invasion distance) was used to determine the invasion ability of L. amazonensis. The transmatrix invasion distance was normalized considering $100 \%$ as the total distance between the top and the bottom of each COL I matrix 
107 histological cut. Similar results were obtained when the data were analyzed without

108 normalization.

109 COL I matrix degradation assay

110 L. amazonensis promastigotes were cultivated inside of 3D COL I matrices prepared with a 111 solution containing 5\% FITC-labeled type I Collagen (Invitrogen, Molecular Probes). The

112 degradation assay was as modified method from Sugiama K. et al., 1980 (Sugiyama et al. 1980).

113 After 0 hours, 24 hours, 48 hours and 72 hours, solid-phase collagens were pelleted (5 min,

$11416,000 \mathrm{~g})$ and the supernatant containing released FITC-labeled type I Collagen fragments was 115 measured with a spectrofluorimeter (485 $\mathrm{nm}$ excitation, $515 \mathrm{~nm}$ emission). The background 116 signal and the total degradation were obtained from a cell-free matrix and from a matrix made 117 with the presence of $25 \mathrm{U} / \mathrm{mL}$ type II collagenase (Gibco) respectively.

\section{Zymography}

119 Promastigotes cultivated for 48 hours inside COL I matrix were placed in an eppendorf tube and 120 centrifuged for $7 \mathrm{~min}$ at $1600 \mathrm{~g}$ to separate the COL I matrix and cells from the supernatant. After 121 centrifugation, supernatant was filtered to remove any remaining cells. Proteins from the 122 supernatant were then separated by SDS-PAGE gel electrophoresis using gels containing 1.5 $123 \mathrm{mg} / \mathrm{mL}$ COL I and 10\% acrylamide and bis-acrylamide. After electrophoresis, gels were washed 124 with $50 \mathrm{mM}$ Tris $2.5 \%$ Triton-X100 buffer (pH 6.8) for $30 \mathrm{~min}$ and then incubated with $50 \mathrm{mM}$ 125 Tris, $10 \mathrm{mM} \mathrm{CaCl}_{2}, 1 \mathrm{mM}$ DTT buffer ( $\mathrm{pH}$ 6.8) for 48 hours. When indicated, the protease 126 inhibitors o-phenantroline $5 \mathrm{mM}$ or E-64 $20 \mu \mathrm{M}$ were added to the reaction buffer.

\section{3D COL matrix model for promastigote-macrophage interaction}


128 The murine macrophages RAW 264.7 cell line $\left(3 \times 10^{5}\right)$ were mixed with $1 \mathrm{~mL}$ of diluted and

129 neutralized COL I solution and incubated at $37^{\circ} \mathrm{C}$ for a 1 hour polymerization. After complete

130 polymerization, RPMI medium complemented with 5\% bovine serum was added to the top of the

131 matrices to feed the cells and hydrate the COL I matrix. The culture was then left at $37^{\circ} \mathrm{C}$ for 24

132 hours. Macrophage 3D culture viability was analyzed using a fluorescent live dye fluorescein

133 diacetate (Sigma). After 24 hours of incubation, the medium was removed and Leishmania

134 promastigotes were add to the $3 \mathrm{D}$ culture in two ways: $10^{7}$ promastigotes in $200 \mu \mathrm{L}$ of RPMI

135 were injected inside the matrix containing macrophages (injection mode) or promastigotes were

136 added to the top of the matrix (invasion mode).

\section{Confocal microscopy}

138 Live promastigotes were labeled with $5 \mathrm{mM}$ of cell tracker green (Invitrogen-C7025) for $15 \mathrm{~min}$.

139 Macrophages cultured in a 3D COL I matrix for 24 hours were than labeled and put to interact

140 with labeled L. amazonensis promastigotes for 4 hours. After interaction the samples were fixed

141 with 4\% paraformaldehyde and than permeabilized with TritonX100 $0.05 \%$ and incubated with

142 rhodamine phalloidin (Invitrogen) overnight at $4{ }^{\circ} \mathrm{C}$. The samples were visualized by the 143 confocal microscopy LEICA TCS SP5 and the images were processed using the software LAS 144 AF.

145 Results

146 L. amazonensis promastigotes extensively interact with and remodel 3D COL I matrix fibers

147 Scanning Electron Microscopy (SEM) of promastigotes cultivated in a 3D COL I matrix showed

148 a huge number of promastigotes extensively adhered to COL I fibers (Fig. 1). The presence of 149 promastigotes altered the organization of the COL I fibers network (Fig. 1B-F). This matrix 150 remodeling transformed the original homogeneous COL I matrix (Fig. 1A) into a meshwork 
151 divided into areas of high fiber density and large fiber-free channels (Fig. 1E, F). Promastigote

152 cell bodies and/or flagella were observed in contact with the COL I fibers (Fig. 1C). SEM

153 revealed promastigotes completely trapped in the high density COL I fiber areas (Fig. 1D) and

154 promastigotes in the fiber-free channels areas. Promastigotes had their cell body elongated over

155 time during the 3D cultures on COL I matrices (S. 1), a feature normally associated with standard 156 liquid cultures.

\section{3D COL I matrix degradation by $L$. amazonensis promastigotes}

158 Promastigotes were cultivated inside 3D FITC-COL I matrices to measure their ability to degrade

159 this network. L. amazonensis promastigotes degraded COL I fibers in a time-dependent manner

160 and density-independent way (Fig. 2A-B). After 72 hours, around 23\% of the COL I total matrix

161 was degraded. This degradation was significantly reduced in the presence of the cysteine protease

162 inhibitors cystatin or E-64 (Fig. 3). The presence of the metalloprotease inhibitor marimastat also

163 reduced COL I degradation, but no effect was observed with the serine protease inhibitor AEBSF

164 (Fig. 3). Incubation with anti-gp63 caused a small but significant reduction of COL I degradation,

165 suggesting that this metalloprotease is also capable of COL I cleavage. COL degradation was not

166 completely abolished in the presence of protease inhibitor (PI) mix (Fig. 3). Although the

167 concentration of inhibitors used could be insufficient for complete inhibition, we chose not to use

168 higher concentrations of this inhibitor mix because they affected cell viability (data not shown).

169 Zymography of culture supernatant from L. amazonensis grown in a COL I matrix detected two

170 collagenase protease bands (S. 2). The collagenase bands completely disappeared in the presence

171 of the metalloprotease inhibitor O-phenanthroline, demonstrating the secretion of COL I-

172 degrading metalloproteases by L. amazonensis promastigotes (S. 2). Other classes of proteases

173 could not be detected by COL zymography in the conditions tested. However, reaction buffers of 
$174 \mathrm{pH}$ lower than 6.8 destroy the COL gels, restricting the use of this assay for Leishmania cysteine

175 proteases.

\section{L. amazonensis migration and transmatrix migration in a 3D COL I matrix}

177 L. amazonensis promastigote migration inside of a 3D COL I matrix was observed by video

178 microscopy (data not shown). These flagellated protozoa displayed an intermittent migration

179 mode with displacement periods (run phase) intercalated with non-displacement periods (freeze

180 phase). The freeze and run migration mode contrasts with the continuous swimming migration

181 mode found in conventional in vitro liquid culture. The migration speed in 3D cultures

182 significantly increased over time (Fig. 4), reaching the highest mean rate $(6 \mu \mathrm{m} / \mathrm{sec})$ after 72

183 hours. Even at the first time point, just after COL I matrix polymerization, promastigotes had a

184 slow but non-zero migration rate (Fig. 4). Over time, the displacement periods were more

185 frequent and lasted longer, reflecting a higher migration speed (Fig. 4). Thus, there was a

186 correlation between time, increased speed, and COL I matrix remodeling by promastigotes.

187 In addition to migration within a 3D COL I matrix, we tested the ability of promastigotes to

188 adhere to and cross the matrix (transmatrix migration). Indeed, when promastigotes were added

189 to the top of a polymerized 3D COL I matrix they were able to adhere to and penetrate through

190 the COL I fiber meshwork (Fig. 5A). This matrix invasion ability (transmatrix migration)

191 demonstrates that promastigotes are not only able to migrate inside of a 3D COL matrix (Fig. 4)

192 but are also able to cross from a liquid environment (RPMI media) to a 3D meshwork (COL I

193 matrix). Here it is important to note that promastigote transmatrix migration was observed

194 without the addition of an external chemo-attractive factor, demonstrating a natural COL I matrix

195 invasive ability for this intracellular parasite. The distance reached by promastigotes from the top

196 of the matrix (transmatrix migration distance) was significantly reduced at higher COL I densities

197 (3.0 mg/ml) compared to lower densities (1.5 mg/ml) (Fig. 5B). 


\section{Extracellular protease activity promotes $L$. amazonensis 3D COL I migration and invasion.}

199 The correlation between protease activity and promastigote migration in 3D was analyzed by

200 videomicroscopy in the presence or absence of a protease inhibitor (PI) cocktail. The 201 promastigote migration rates were analyzed using the software ImageJ. In the presence of a PI 202 mix composed of E-64, Marimastat and Cystatin, the promastigote migration rates inside the 3D 203 COL I matrix were significantly reduced, demonstrating that protease activity increases migration 204 rates (Fig. 6). The fraction of promastigotes with migration rates exceeding $5 \mu \mathrm{m} / \mathrm{second}$ was also 205 significantly decreased by the PI mix (Fisher's exact test, Fig. 6). The reduction of migration 206 rates could also be observed when only the run phase was considered in the analysis, indicating 207 an inhibition of both frequency and maximum speed of migration.

208 In addition, the role of protease activities in promastigote transmatrix migration was evaluated 209 after 72 hours, in the presence or absence of PI mix (Fig. 7). Transmatrix migration is dependent 210 on two processes: the ability of cells to penetrate into the matrix and, once inside, the ability to 211 migrate. The PI mix presence reduced by almost $40 \%$ the number of promastigotes inside of the 212 COL I matrix (Fig. 7A) and also significantly reduced the maximal transmatrix migration 213 distance (in depth) achieved by the promastigotes (Fig. 7B).

214 Together the data demonstrate that extracellular protease activity is important for L. amazonensis 215 promastigote transmatrix and migration in 3D cultures (Fig. 6-7), due to COL I matrix 216 degradation/remodeling.

\section{Interaction of macrophages and promastigotes in 3D COL I matrix}

218 For an even better mimic of what would happen during Leishmania infection, we cultivated 219 macrophages inside the COL I matrix for 24 hours, after which L. amazonensis promastigotes 220 were added to the top of the matrix (Fig. 8). After 6 hours of interaction most of the parasites 
221 were found inside the matrix (Fig. 8), a shorter time than in the macrophage-free matrix. This

222 suggests an influence of macrophages on the invasion ability of Leishmania promastigotes. 3D

223 Visualization by confocal microscopy of macrophage-promastigote interaction (Fig. 9A-B)

224 showed macrophages possessing a round morphology with many actin-membrane filament

225 projections (Fig. 9C). In some cases these thin actin-membrane projections were touching a

226 promastigote's surface (Fig. 9D). SEM microscopy observations showed the extensive contact of

227 macrophages and promastigotes with COL I fibers in the 3D culture model (Fig. 10). The fibrotic

228 characteristic of the ECM 3D environment seems to create a effective barrier for physical contact

229 between Leishmania and macrophages, in contrast to 2D culture models in which macrophages

230 become infected much earlier.

231 The injection of promastigotes directly into the matrix containing macrophages allowed a good

232 distribution of promastigotes inside the COL meshwork and enabled the observation of 233 macrophage-promastigote interaction in the first minutes of interaction (data not shown). That

234 result demonstrated the promastigotes migrating further and faster than macrophages in the 3D 235 culture. Additionally, the promastigotes exhibited the same freeze and run migration mode 236 observed in the macrophage-free 3D matrix.

\section{Discussion}

238 In the human host, Leishmania amazonensis is an intracellular parasite that mainly proliferates 239 inside macrophages. Therefore, the interaction of the Leishmania parasite with the host ECM has

240 been neglected. In fact, it is critical to understand the interaction of Leishmania promastigotes

241 with the ECM because when a host is infected, the promastigotes must pass through the dermis

242 ECM, remaining there until the first contact with macrophages (Lira et al. 1997; McGwire et al.

2432003 ) or other potential host cells. Our results demonstrated that L. amazonensis promastigotes

244 extensively contacted the COL I fibers in a 3D matrix (Fig.1D). Following these interactions 
245 were drastic modifications of the COL I fiber organization (Fig. 1A-F). Many publications have

246 shown a correlation between ECM remodeling and inflammation, and indeed, abnormal ECM

247 organization is prominent in many diseases such as tissue fibrosis and cancer (Cox \& Erler 2011).

248 This suggests that COL I matrix remodeling by promastigotes could play an important role during

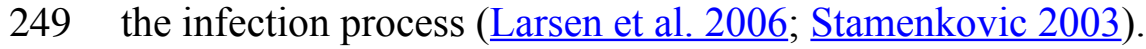

$2503 \mathrm{D}$ culture is becoming a common approach for the study of several types of mammalian cells

251 (Voytik-Harbin 2001) but it is still not commonly used for pathogens or unicellular organisms

252 (Behnsen et al. 2007). In this work we establish, for the first time, 3D in vitro cultivation of $L$.

253 amazonensis promastigotes. This in vitro 3D culture mimics (at least in part) the physical

254 dynamics of the Leishmania human host environment and makes possible the in vitro study of the

255 mechanisms involved in the interaction of L. amazonensis with the ECM. Conventionally,

256 promastigotes are cultivated in rich serum-containing media to maintain $90 \%$ viability. However,

257 in our model, L. amazonensis promastigotes were able to survive ( $>90 \%$ viability) and proliferate

258 for 72 hours inside a 3D matrix composed of 1.5 or $3.0 \mathrm{mg} / \mathrm{mL}$ of COL I in serum-free RPMI

259 medium, suggesting that this flagellated parasite, like some mammalian cells, required fewer

260 survival signals when in 3D culture (Alavi \& Stupack 2007; Eke \& Cordes 2011). This could be

261 due to the physical support presented in the 3D matrix or to the chemical signaling provided by

262 the COL I molecules (Weaver et al. 2002).

263 The roles that Leishmania proteases play during inflammation and interaction with macrophages

264 are important for infection, especially in the case of the metalloprotease gp63 (Silva-Almeida et

265 al. 2012b; Yao 2010). Here, we presented a new role for extracellular Leishmania proteases.

266 Protease blockage by a mixture of inhibitors decreased matrix degradation and affected

267 promastigote migration and transmigration among COL I fibers. The ability of promastigotes to

268 cleave COL I matrix fibers was analyzed by a degradation assay using fluorescent COL I. We

269 showed that promastigotes can actively degrade COL I fibers and that the degradation was 
270 independent of the matrix density (Fig. 2). Metallo- and cysteine proteases were implicated in

271 this degradation (Fig. 3). The presence of anti-gp63 significantly reduced the COL I degradation,

272 indicating the importance of this protease in the cleavage of COL I fibers. However, the presence

273 of the PI mix did not completely abolish the COL I degradation. These findings suggest that

274 either the inhibitors are not strong enough to block all the extracellular proteases without

275 affecting the parasite survival, or that the mechanical pushing and pulling by the promastigote

276 flagella also contribute to COL I fiber degradation.

277 Following the promastigotes by videomicroscopy, we showed that these parasites were able to

278 migrate inside the 3D COL I matrix (Fig. 4). However, the migration mode in this 3D culture was

279 completely different from the one in standard culture medium. In the 3D culture environment, the

280 promastigote migration oscillates between periods of movement and stationary periods, which we

281 described as a freeze and run migration mode (data not shown). This data is consistent with our

282 SEM data (Fig.1), showing some promastigotes completely trapped in the COL I fibers (freeze

283 phase) and others COL I fiber-free (run phase), probably passing through the COL I matrix

284 tunnels created by matrix remodeling (Fig.1). The migration rate and COL I matrix degradation

285 both increased over time (Fig. 2-4), consistent with the hypothesis that the run phase of migration

286 is linked to the presence of COL I matrix tunnels.

287 In the presence of PI mix the rate of promastigote migration inside the matrix was significantly

288 reduced (Fig. 6). This reduction was more pronounced when only the run phase was considered;

289 the maximum speed reached in that phase was significantly slower in the presence of PIs (Fig. 6).

290 These results suggest that the extracellular Leishmania proteases and the subsequent COL I

291 matrix remodeling lead directly to an increased migration rate.

292 Transmatrix migration is an important skill of metastatic cells, invasive extracellular parasites,

293 and of immune cells such as neutrophils and macrophages (Hagedorn \& Sherwood 2011;

294 Schoumacher et al. 2011). L. amazonensis promastigotes were able to invade matrices of multiple 
295 COL I densities (Fig. 5), showing that intracellular parasites possess the ability not only to

296 migrate inside of a COL I matrix but also to adhere to and invade these matrices (transmatrix

297 migration), raising the unexpected possibility of invasive behavior by this intracellular parasite

298 during infection. It is important to mention that all our experiments were made with stationary

299 phase promastigotes (Methods) not enriched for metacyclics and we do not have access to the

300 metacyclic / procyclic proportion of our promastigote culture. Therefore, it is possible that these

301 forms have different behavior in 3D cultures and further work is needed to evaluate their roles.

302 Taken together, we showed that promastigotes degrade COL I matrix via extracellular cysteine

303 and metalloprotease, and these proteases play a role during migration and invasion of 3D

304 matrices. Together these results suggest that the matrix remodeling contributes to L. amazonensis

305 migration and invasion by helping promastigotes to get free from the COL I matrix traps

306 (reducing the freeze period), or even to open the tunnels inside of the matrix that enable a faster

307 maximum migration speed. In both ways, protease release and the subsequent matrix remodeling

308 could help the L. amazonensis promatigotes to create a 3D environment that facilitates access to

309 their intracellular "home".

310 Furthermore, we establish a 3D co-culture model with macrophages seeded in a COL I matrix for

31124 hours before the addition of L. amazonensis promastigotes. The promastigotes were added in

312 two ways: at the top of the matrix (invasion model) or by syringe injection directly inside the

313 matrix. Both models resulted in direct Leishmania-macrophage contact inside the 3D

314 environment. However, the invasion model was necessary for the analysis of the trans-matrix

315 migration of promastigotes during interaction (Fig. 8), while the injection model created a more

316 homogenous distribution of promastigotes in the matrix, allowing videomicroscopy of

317 Leishmania-macrophages interactions (data not shown). Surprisingly, promastigotes migrated

318 faster than macrophages in this model, supporting an active participation of promastigotes during

319 Leishmania infection. The Leishmania-macrophage 3D interaction assay established here will 
320 help us to better understand some aspects of Leishmania-macrophage and Leishmania-matrix

321 interaction and could become an important model for initial drug screens.

\section{Acknowledgements}

323 We thank Dr. Sherri Smith and Dr. Robin C. Friedman for helpful comments on the manuscript.

\section{Abbreviations}

325 COL I: Collagen I

326 3D: three-dimensional

327 ECM: extracellular matrix

328 FITC: fluorescein isothiocyanate

329 PI: protease inhibitors

330 SEM: scanning electron microscopy 
Abreu-Silva AL, Calabrese KS, Mortara RA, Tedesco RC, Cardoso FO, Carvalho LO, and Goncalves da Costa SC. 2004. Extracellular matrix alterations in experimental murine Leishmania (L.) amazonensis infection. Parasitology 128:385-390.

Alavi A, and Stupack DG. 2007. Cell survival in a three-dimensional matrix. Methods Enzymol 426:85-101.

Barral A, Pedral-Sampaio D, Grimaldi Junior G, Momen H, McMahon-Pratt D, Ribeiro de Jesus A, Almeida R, Badaro R, Barral-Netto M, Carvalho EM, and Johnson WD. 1991. Leishmaniasis in Bahia, Brazil: evidence that Leishmania amazonensis produces a wide spectrum of clinical disease. Am J Trop Med Hyg 44:536546.

Behnsen J, Narang P, Hasenberg M, Gunzer F, Bilitewski U, Klippel N, Rohde M, Brock M, Brakhage AA, and Gunzer M. 2007. Environmental dimensionality controls the interaction of phagocytes with the pathogenic fungi Aspergillus fumigatus and Candida albicans. PLoS Pathog 3:e13.

Chang KP, and McGwire BS. 2002. Molecular determinants and regulation of Leishmania virulence. Kinetoplastid Biol Dis 1:1.

Cox TR, and Erler JT. 2011. Remodeling and homeostasis of the extracellular matrix: implications for fibrotic diseases and cancer. Dis Model Mech 4:165-178.

Eke I, and Cordes N. 2011. Radiobiology goes 3D: how ECM and cell morphology impact on cell survival after irradiation. Radiother Oncol 99:271-278.

Hagedorn EJ, and Sherwood DR. 2011. Cell invasion through basement membrane: the anchor cell breaches the barrier. Curr Opin Cell Biol 23:589-596.

Hynes RO. 2009. The extracellular matrix: not just pretty fibrils. Science 326:1216-1219.

Kaye P, and Scott P. 2011. Leishmaniasis: complexity at the host-pathogen interface. Nat Rev Microbiol 9:604-615.

Kulkarni MM, Jones EA, McMaster WR, and McGwire BS. 2008. Fibronectin binding and proteolytic degradation by Leishmania and effects on macrophage activation. Infect Immun 76:1738-1747.

Larsen M, Artym VV, Green JA, and Yamada KM. 2006. The matrix reorganized: extracellular matrix remodeling and integrin signaling. Curr Opin Cell Biol 18:463-471.

Lira R, Rosales-Encina JL, and Arguello C. 1997. Leishmania mexicana: binding of promastigotes to type I collagen. Exp Parasitol 85:149-157.

McGwire BS, Chang KP, and Engman DM. 2003. Migration through the extracellular matrix by the parasitic protozoan Leishmania is enhanced by surface metalloprotease gp63. Infect Immun 71:1008-1010.

McMaster WR, Morrison CJ, MacDonald MH, and Joshi PB. 1994. Mutational and functional analysis of the Leishmania surface metalloproteinase GP63: similarities to matrix metalloproteinases. Parasitology 108 Suppl:S29-36.

Melo FA, Moura EP, Ribeiro RR, Alves CF, Caliari MV, Tafuri WL, Calabrese KS, and Tafuri WL. 2009. Hepatic extracellular matrix alterations in dogs naturally infected with Leishmania (Leishmania) chagasi. Int $J$ Exp Pathol 90:538-548.

Murray HW, Berman JD, Davies CR, and Saravia NG. 2005. Advances in leishmaniasis. Lancet 366:1561-1577.

Neuber H. 2008. Leishmaniasis. J Dtsch Dermatol Ges 6:754-765.

Rogers ME. 2012. The Role of Leishmania Proteophosphoglycans in Sand Fly Transmission and Infection of the Mammalian Host. Front Microbiol 3:223.

Schoumacher M, Louvard D, and Vignjevic D. 2011. Cytoskeleton networks in basement membrane transmigration. Eur J Cell Biol 90:93-99.

Silva-Almeida M, Carvalho LO, Abreu-Silva AL, Souza CS, Hardoim DJ, and Calabrese KS. 2012a. Extracellular matrix alterations in experimental Leishmania amazonensis infection in susceptible and resistant mice. Vet Res 43:10.

Silva-Almeida M, Pereira BA, Ribeiro-Guimaraes ML, and Alves CR. 2012b. Proteinases as virulence factors in Leishmania spp. infection in mammals. Parasit Vectors 5:160.

Stamenkovic I. 2003. Extracellular matrix remodelling: the role of matrix metalloproteinases. J Pathol 200:448-464.

Sugiyama K, Yamamoto K, Kamata O, and Katsuda N. 1980. A simple and rapid assay for collagenase activity using fluorescence-labeled substrate. Kurume Med J 27:63-69.

Voytik-Harbin SL. 2001. Three-dimensional extracellular matrix substrates for cell culture. Methods Cell Biol 63:561-581.

Weaver VM, Lelievre S, Lakins JN, Chrenek MA, Jones JC, Giancotti F, Werb Z, and Bissell MJ. 2002. beta4 integrin-dependent formation of polarized three-dimensional architecture confers resistance to apoptosis in normal and malignant mammary epithelium. Cancer Cell 2:205-216.

Yao C. 2010. Major surface protease of trypanosomatids: one size fits all? Infect Immun 78:22-31. 
387 Yao C, Leidal KG, Brittingham A, Tarr DE, Donelson JE, and Wilson ME. 2002. Biosynthesis of the major surface 388 protease GP63 of Leishmania chagasi. Mol Biochem Parasitol 121:119-128. 


\section{Figure 1}

SEM analysis of $L$. amazonensis interaction with COL I matrices

SEM of normal COL I fiber organization in the 3D matrix without promastigotes $(\mathrm{A})$ and promastigotes interacting with $\mathrm{COL}$ I fibers of a 3D matrix (B-F). Promastigotes extensive alter $\mathrm{COL}$ I fiber organization (B-D). In some matrix areas the L. amazonensis promastigotes were completely trapped between $\mathrm{COL}$ matrix fibers $(\mathrm{E})$. L. amazonensis promastigotes were able to interact with $\mathrm{COL}$ I fibers through their cell bodies and flagella $(\mathrm{C})$. 


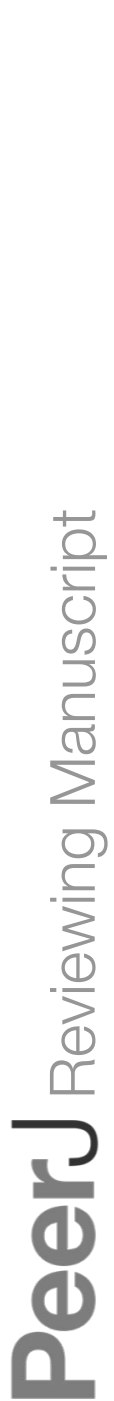

acy r.

B

201 (n)

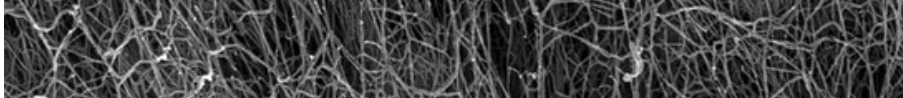
1.2.

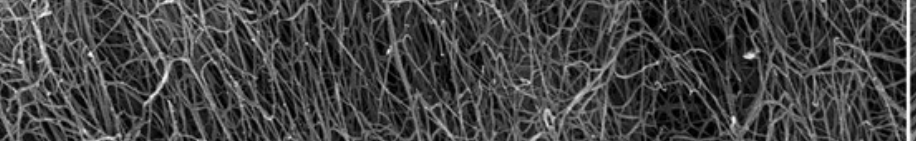

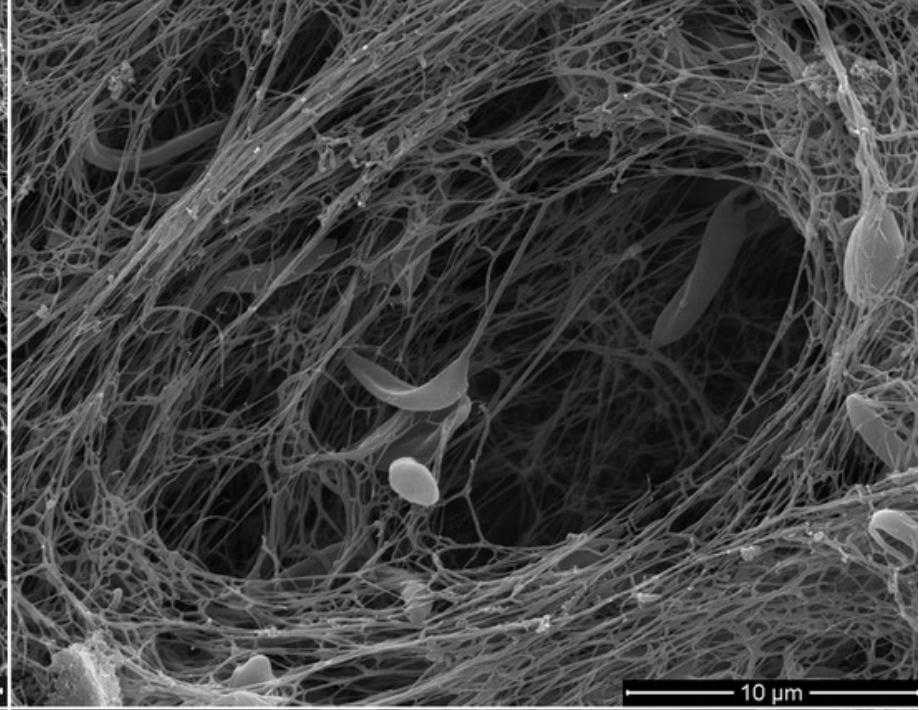

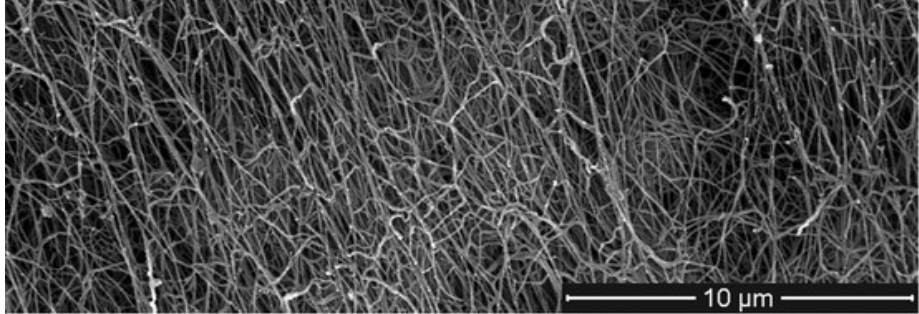

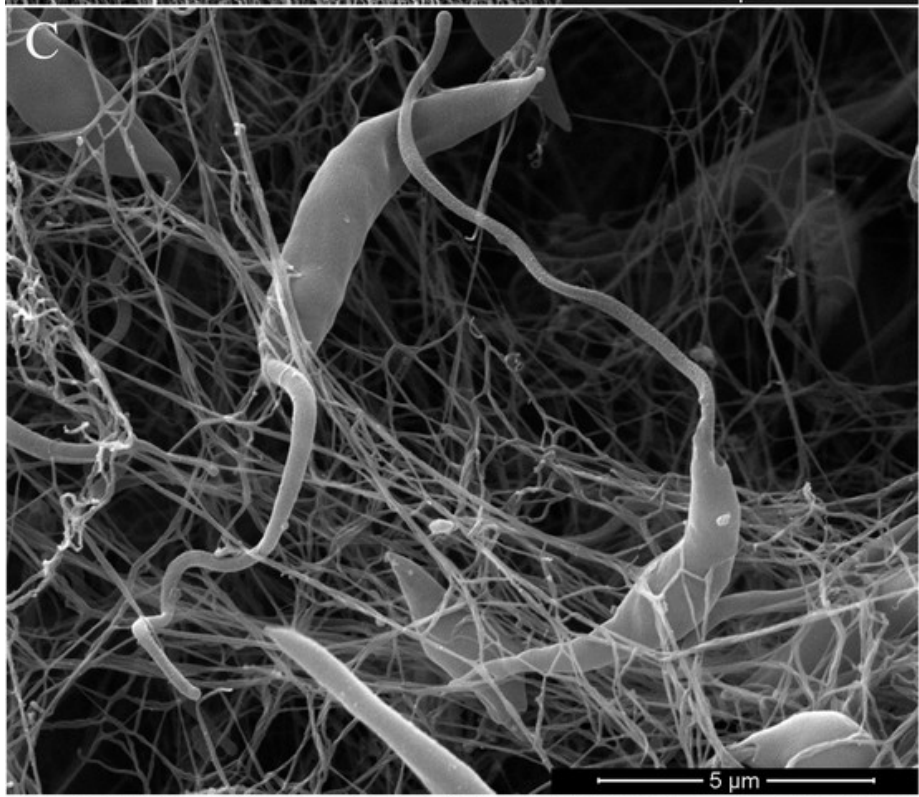

$\mathrm{D}$
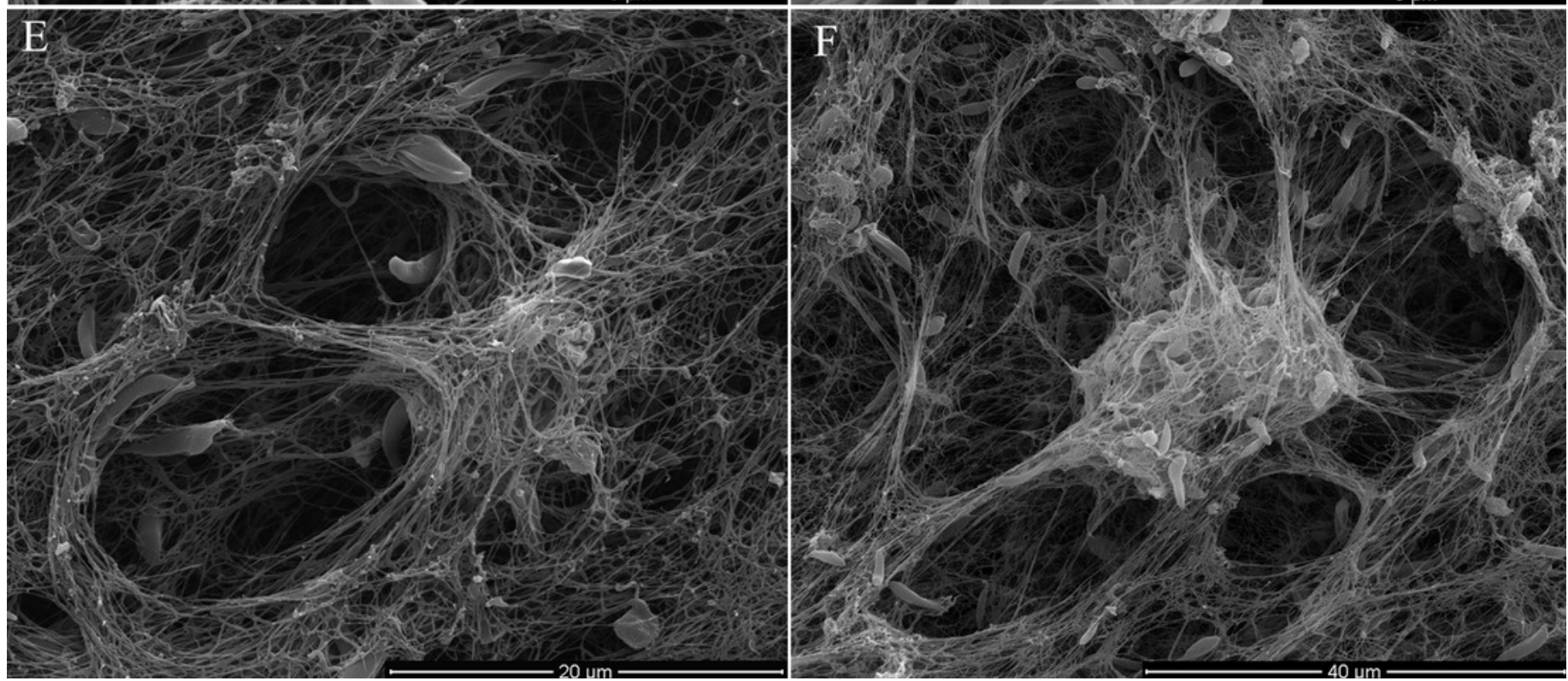


\section{Figure 2}

Collagen matrix degradation by L. amazonensis promastigotes.

COL I matrix degradation was evaluated by the amount of COL I-FITC found in the supernatant after different periods of interaction of $L$. amazonensis promastigotes with COL I matrix $(1.5 \mathrm{mg} / \mathrm{ml})(A)$ or after $72 \mathrm{~h}$ of interaction on low $(1.5 \mathrm{mg} / \mathrm{ml})$ or high $(3.0 \mathrm{mg} / \mathrm{ml}) \mathrm{COL} \mathrm{I}$ matrix density (B). Data are expressed as the mean with whiskers representing the standard error of the mean. $T$ test with Bonferoni's multiple test correction ${ }^{* * *}=p<0.0001,{ }^{* *}=p<$ 0.001 .
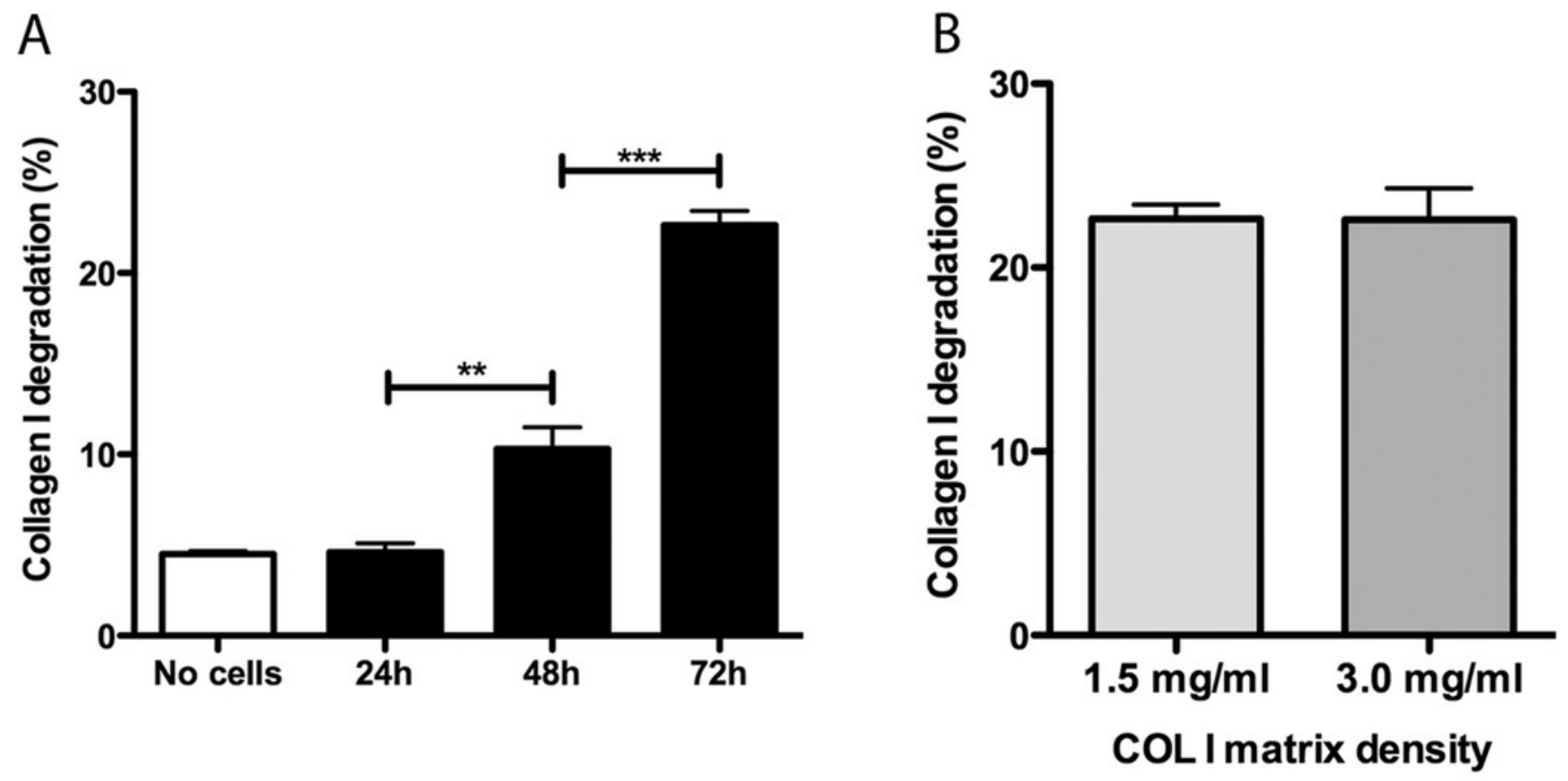


\section{Figure 3}

L. amazonensis promastigotes degrade COL I fiber by protease release.

Promastigote interaction with the COL I matrix occurred in the presence of different proteases inhibitors (PI) and PI mix. Cysteine PI, E-64 (100 $\mu \mathrm{M})$ and cystatin $(20 \mathrm{ng} / \mathrm{mL})$, caused a significant reduction of COL I degradation, however, the metalloprotease inhibitor marimastat $(200 \mathrm{nM})$ caused an even higher reduction. The presence of anti GP-63 (1:50) also reduced COL I degradation ability. Serine protease inhibitor AEBSF (1mM) had no effect on the degradation. Whiskers represent the standard error of the mean. 1-way ANOVA, Kruskal-Wallis test, ${ }^{*}=p<0.05,{ }^{* * *}=p .<0.0001$.

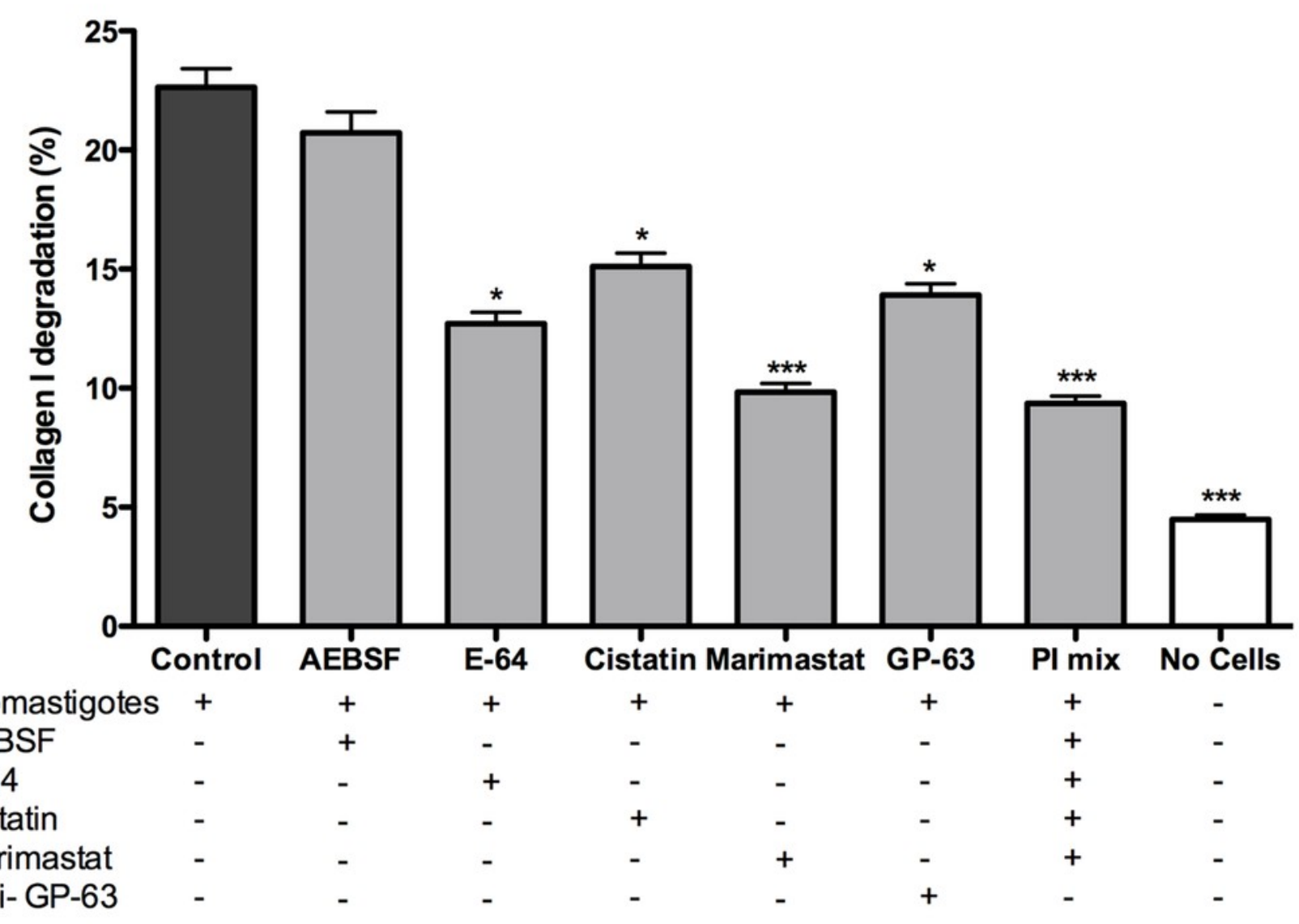




\section{Figure 4}

3D migration rate increases over the time in COL I matrix

L. amazonensis promastigote migration rate inside of COL I 3D matrix over time. The migration was observed by videomicroscopy and the rate analyzed by ImageJ software. Bars represent the standard error of the mean. 1-way ANOVA, ${ }^{* \star}=p<0.001,{ }^{* \star *}=p<0.0001$.

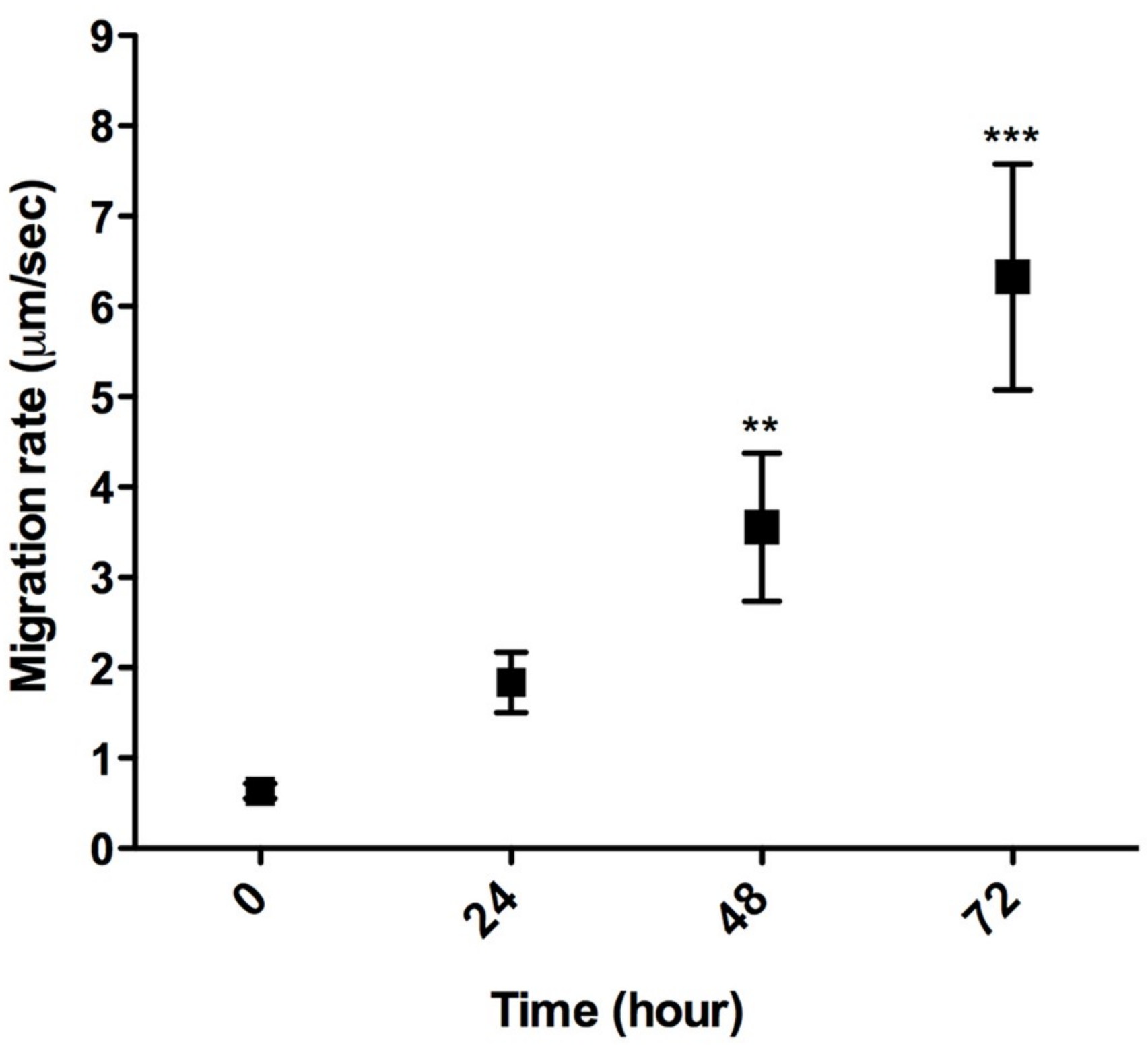




\section{Figure 5}

Trans-matrix migration ability is dependent on the COL I matrix density.

Histological transversal cuts of COL I 3D matrix at 1.5 or $3.0 \mathrm{mg} / \mathrm{mL}$ after $72 \mathrm{~h}$ of $L$.

amazonensis promastigote (arrows) invasion (A). Promastigote $\mathrm{COL}$ I transmatrix migration was measured by the depth reached by the promastigotes divided by the total size of each histological transversal cut (B). Whiskers represent the standard error of the mean. Student's $T_{\text {test }}{ }^{* \star *}=p<0.0001$.

A

Low density matrix (COL I 1.5 mg/ml)

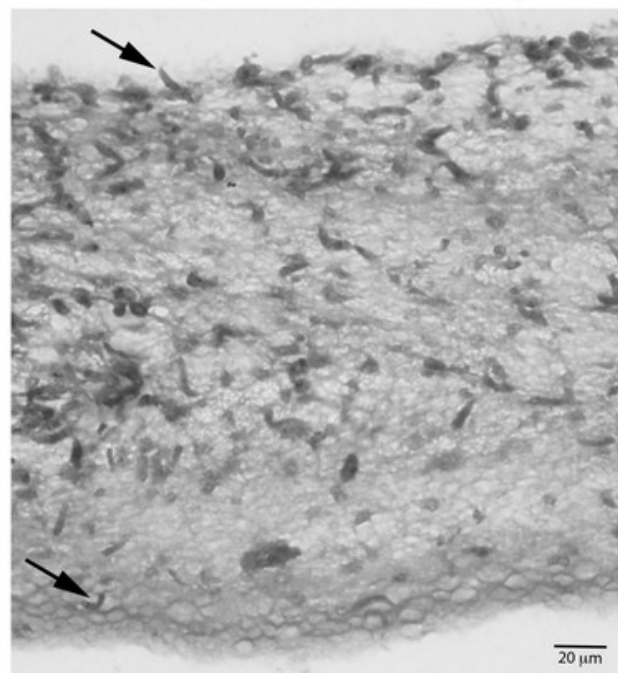

High density matrix (COL I $3.0 \mathrm{mg} / \mathrm{ml})$

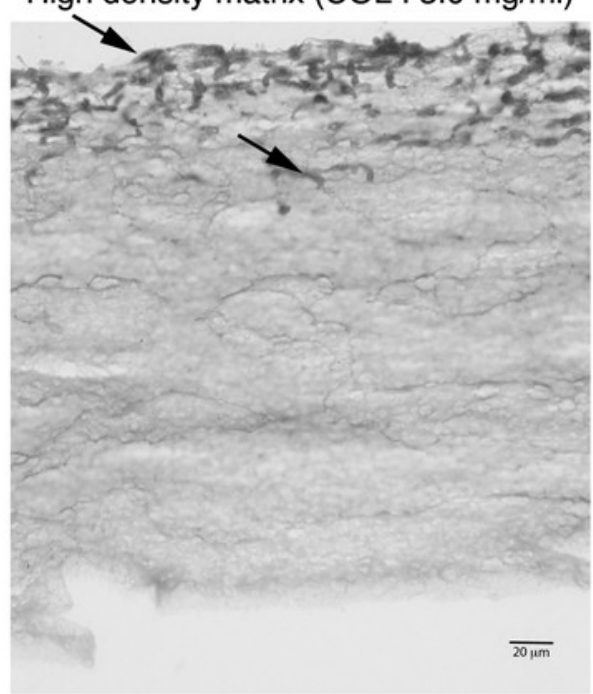

B

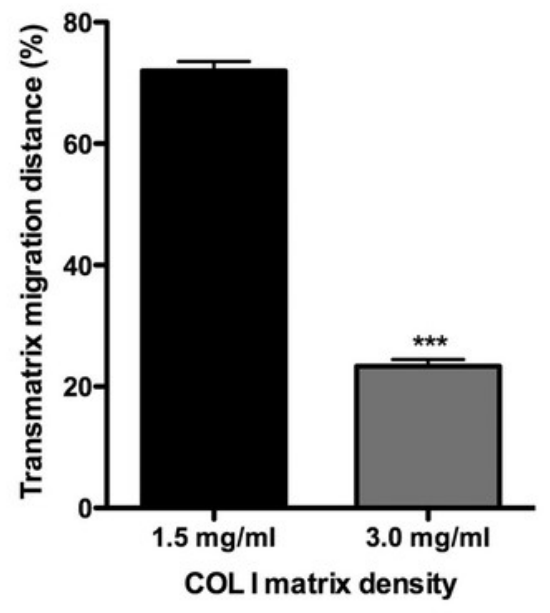




\section{Figure 6}

L. amazonensis migration in 3D cultures with and without protease inhibitors

L. amazonensis promastigote migration rate after 24 hours inside of COL I 3D matrix with or without the protease inhibitors (PI) mix. The migration was observed by videomicroscopy and analyzed by ImageJ software. The migration rate was analyzed in two ways: total migration (freeze and run phases) or during the moving phase of the migration only (run phase). Whiskers represent the minimum and maximum values. Differences in the mean value were tested using Mann-Whitney test ${ }^{* *} p=0.0074$ and $p=0.0019 ; n=45$. Fisher's exact test was used to test differences in the fraction of promastigotes with migration speed greater than 5 $\mu \mathrm{m} / \mathrm{sec}, \mathrm{p}=0.0124$ (Freeze and run phases) and $\mathrm{p}=0.0031$ (run phase). 


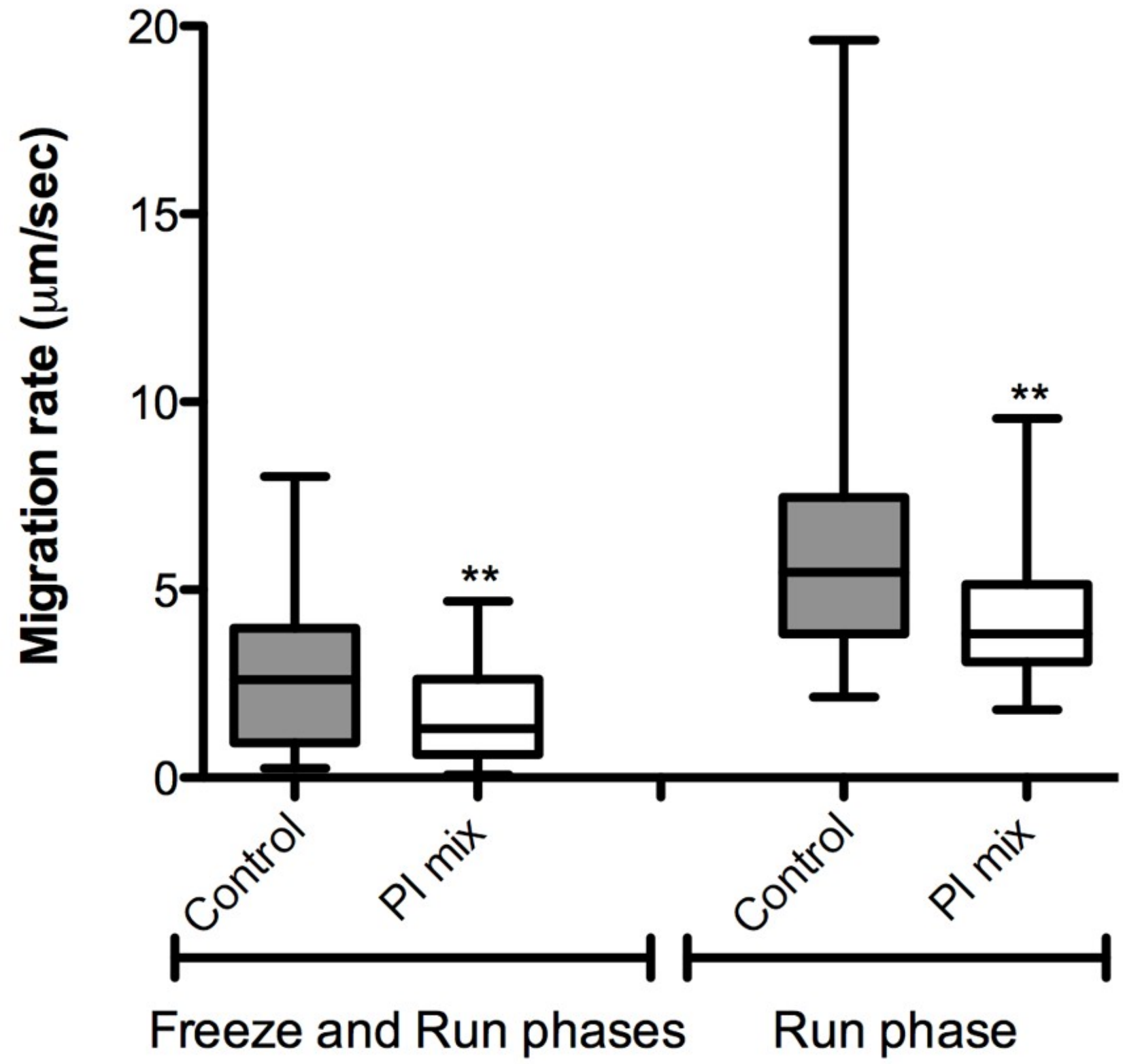




\section{Figure 7}

Trans-matrix migration ability is affected by the presence of a protease inhibitors (PI) mix.

Leishmania promastigotes were add to the top of COL I matrix of $1.5 \mathrm{mg} / \mathrm{ml}$ and the transmatrix invasion ability in presence or absence of protease inhibitors was analyze by the percentage of parasites inside the matrix $(A)$ and by the maximum distance (in deep) achieved by the parasites (B). In both cases the presence of protease inhibitors mix significantly reduced the transmatrix migration ability.
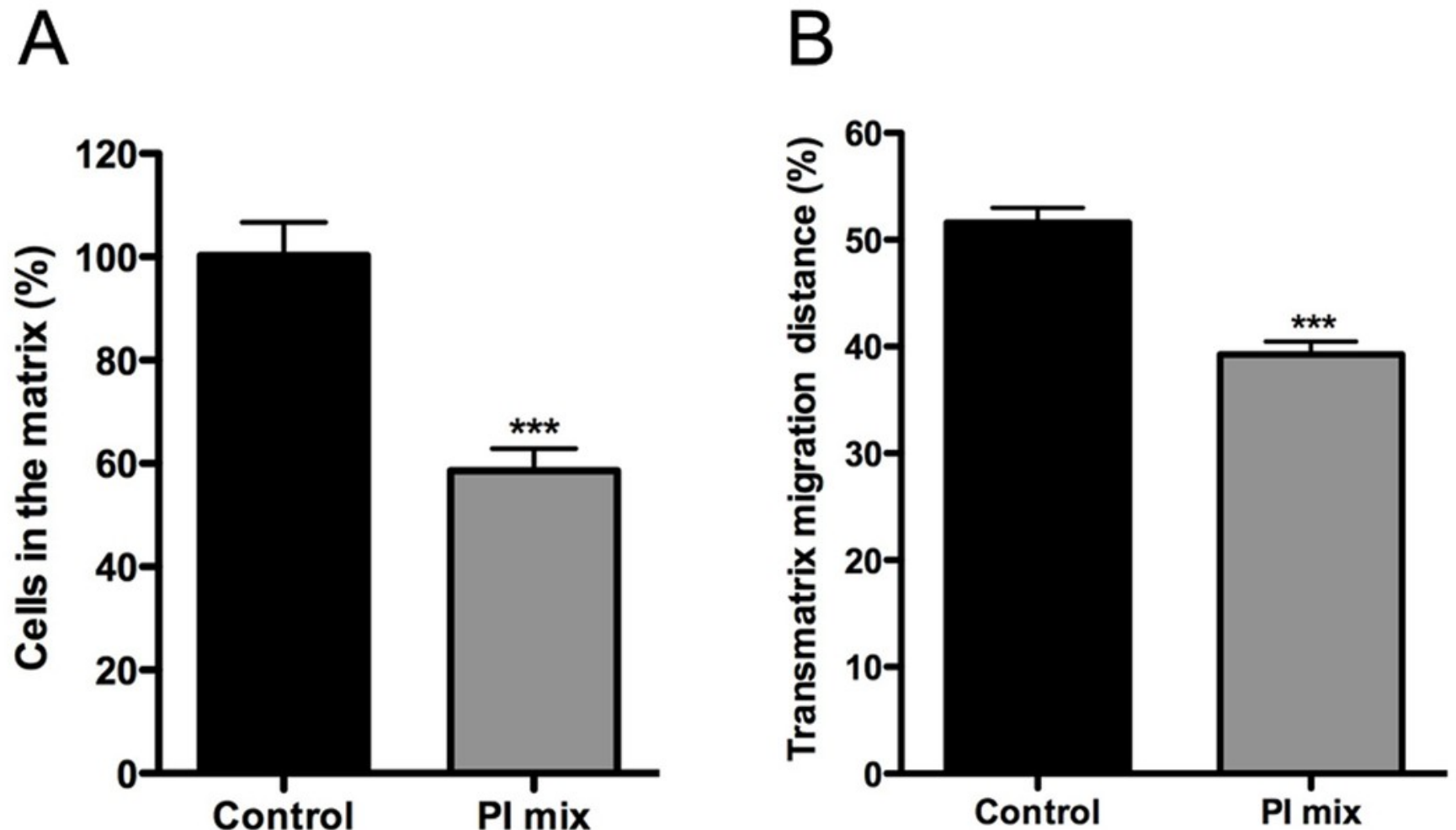


\section{Figure 8}

Promastigote trans-matrix migration ability in the presence of macrophages

Leishmania promastigotes were added to the top of the macrophages 3D COL I culture. After $6 \mathrm{~h}$ the samples were fixed, prepared for histology and transversally cut. Image shows transversal cuts of COL I matrix containing macrophages (red arrows) 6h after addition of $L$. amazonensis (blue arrows) promastigotes (A-B) demonstrating many parasites able to invade the COL I matrix.
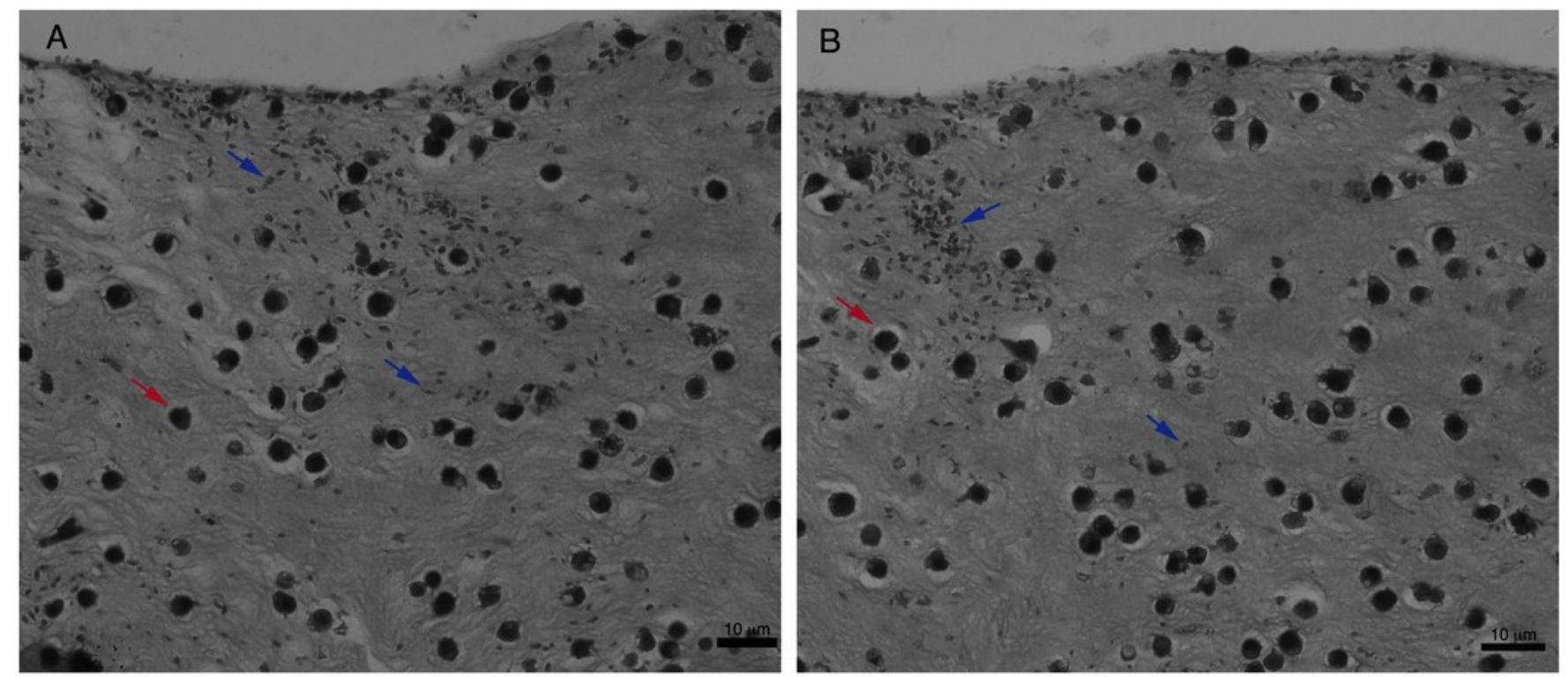


\section{Figure 9}

Macrophage-Leishmania 3D interaction vizualization by confocal microscopy

Leishmania interaction with macrophages in the 3D COLI matrix was visualized by confocal microscopy. Live Leishmania promastigotes were labeled with cell tracker green before interaction. Macrophages actin filaments were stained with phalloidin (Red). Images are 3D reconstruction of a $11 \mu \mathrm{m}$ deep image.
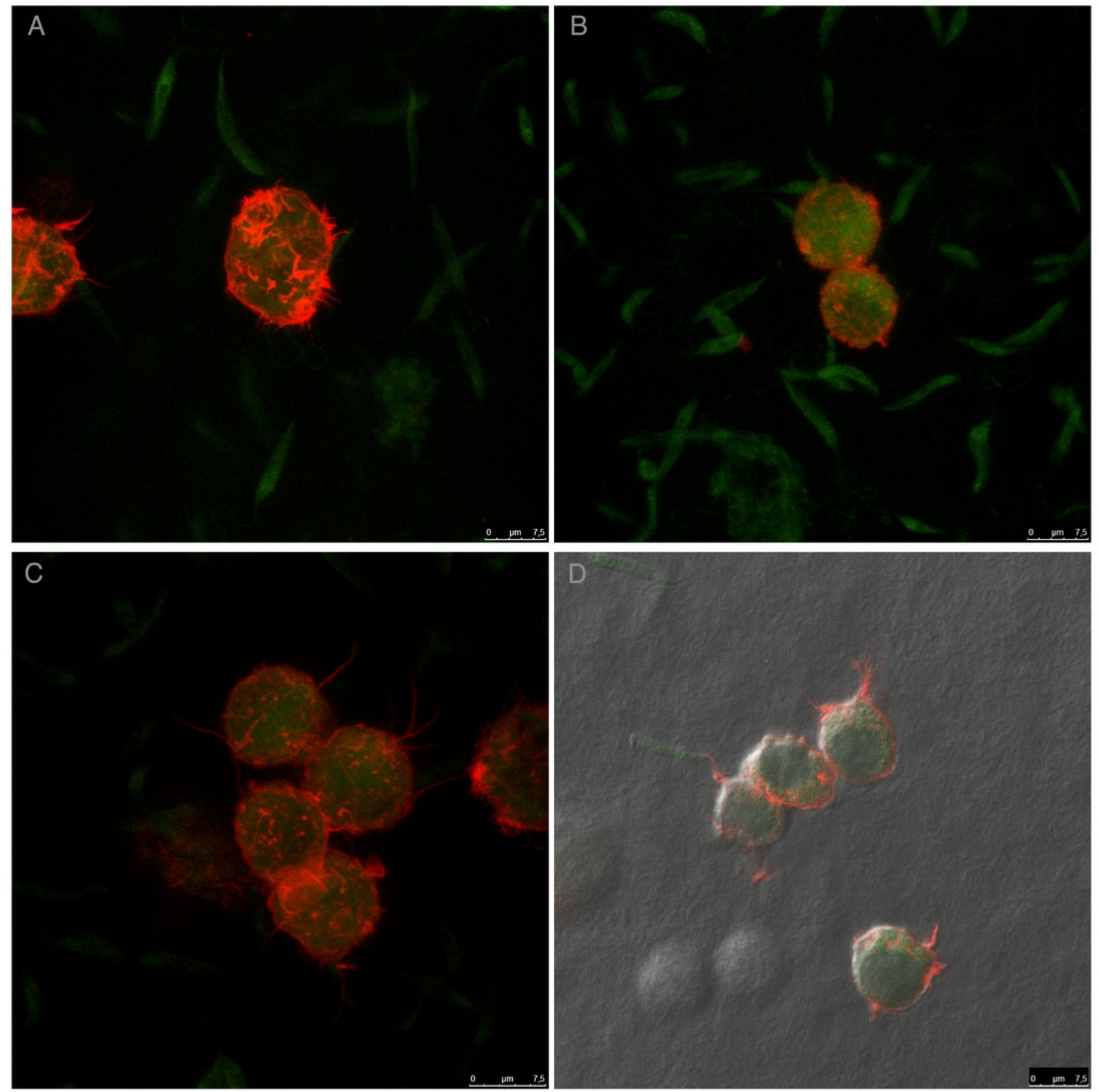


\section{Figure 10}

SEM of L. amazonensis-macrophages 3D interaction

SEM visualization of macrophages cultured in a 3D COL I matrix after $3 \mathrm{~h}(\mathrm{~A})$ or $4 \mathrm{~h}(\mathrm{~B})$ of interaction with L.amazonensis promastigotes. In order to visualize the promastigotemacrophage interaction inside the matrix the samples were transversally cut in liquid nitrogen during SEM samples processing
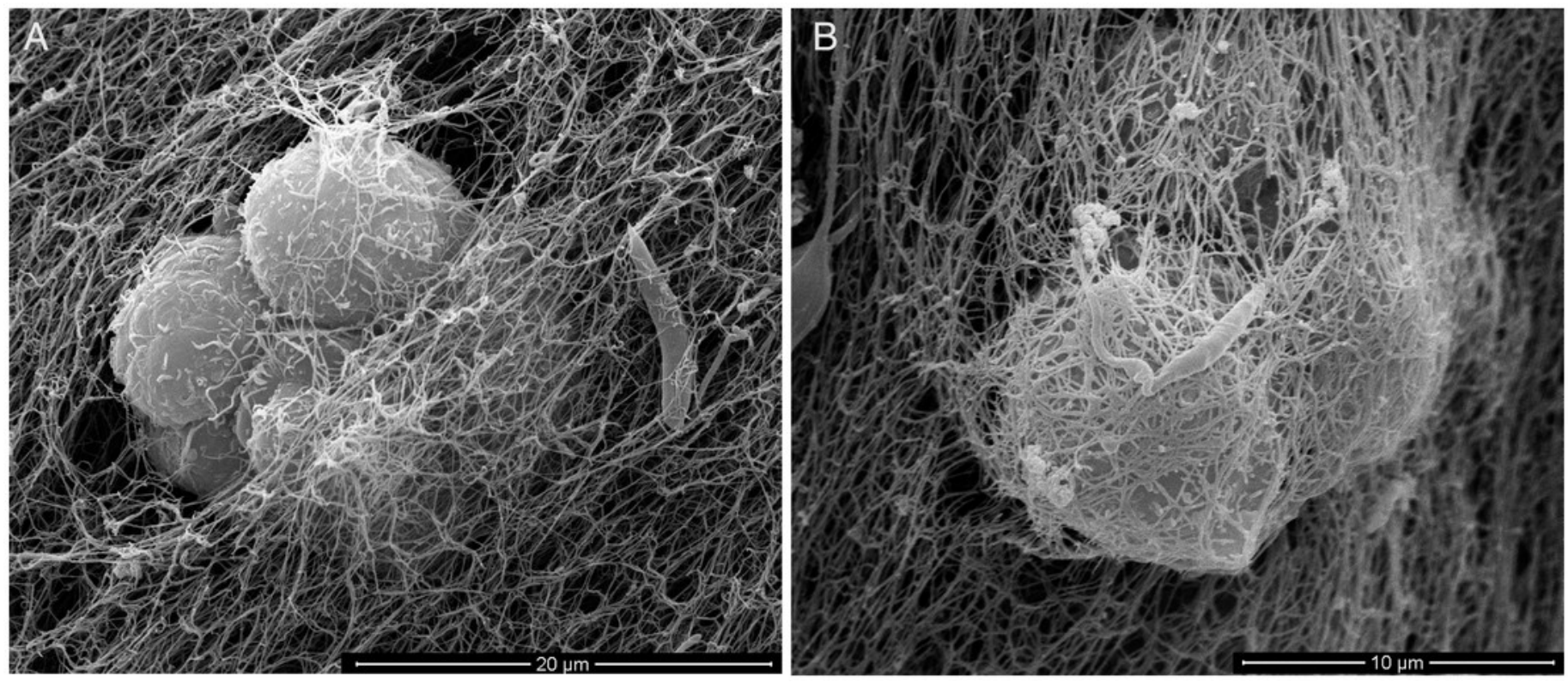